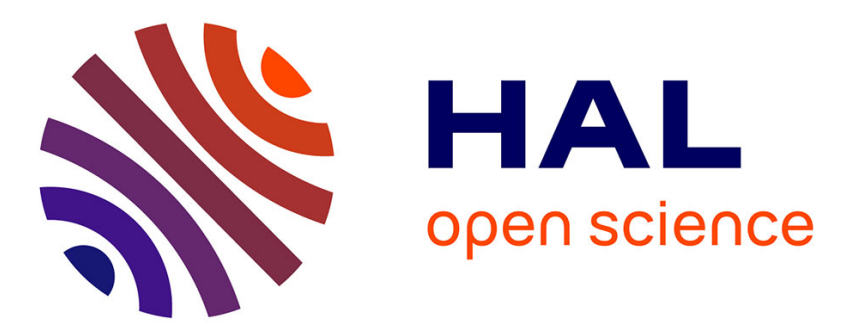

\title{
Homogénéité d'épaisseur et propriétés électriques des couches minces de silice obtenues dans un mélange $\mathrm{N} 2 / \mathrm{O} 2 / \mathrm{HCl}$
}

Gérard Sarrabayrouse, J. Capilla

\section{- To cite this version:}

Gérard Sarrabayrouse, J. Capilla. Homogénéité d'épaisseur et propriétés électriques des couches minces de silice obtenues dans un mélange N2/O2/HCl. Revue de Physique Appliquée, 1985, 20 (2), pp.87-91. 10.1051/rphysap:0198500200208700 . jpa-00245310

\author{
HAL Id: jpa-00245310 \\ https://hal.science/jpa-00245310
}

Submitted on 1 Jan 1985

HAL is a multi-disciplinary open access archive for the deposit and dissemination of scientific research documents, whether they are published or not. The documents may come from teaching and research institutions in France or abroad, or from public or private research centers.
L'archive ouverte pluridisciplinaire HAL, est destinée au dépôt et à la diffusion de documents scientifiques de niveau recherche, publiés ou non, émanant des établissements d'enseignement et de recherche français ou étrangers, des laboratoires publics ou privés. 


\title{
Homogénéité d'épaisseur et propriétés électriques des couches minces de silice obtenues dans un mélange $\mathrm{N}_{2} / \mathrm{O}_{2} / \mathbf{H C l}\left({ }^{*}\right)$
}

\author{
G. Sarrabayrouse et J. Capilla \\ Laboratoire d'Automatique et d'Analyse des Systèmes du Centre National de la Recherche Scientifique, \\ 7, avenue du Colonel Roche, 31077 Toulouse Cedex, France
}

(Reçu le 16 mai 1984, révisé le 8 octobre, accepté le 25 octobre 1984)

\begin{abstract}
Résumé. - L'homogénéité de l'épaisseur et les propriétés électriques de couches de silice obtenues sous pression partielle dans un mélange $\mathrm{O}_{2} / \mathrm{HCl} / \mathrm{N}_{2}$ sont examinées. La présence de $\mathrm{HCl}$ accroît l'homogénéité des caractéristiques électriques.
\end{abstract}

\begin{abstract}
The homogeneity over the wafer of the thickness of very thin silica layers obtained under low partial pressure in a mixture $\mathrm{O}_{2} / \mathrm{HCl} / \mathrm{N}_{2}$ as well as the electrical properties of the layers are examined. It is shown that the chlorine content increases the homogeneity of the thickness and the reproductibility of the electrical properties.
\end{abstract}

\section{Introduction.}

Les propriétés électriques des couches très fines de silice ont fait l'objet de nombreux travaux et leur intérêt s'est accru récemment du fait d'applications aux mémoires EEPROM [1] pour les couches d'épaisseurs voisines de $100 \AA$, aux dispositifs MISS [2] aux mémoires MNOS [3] ou aux cellules solaires [4] en ce qui concerne les couches d'épaisseurs inférieures à $50 \AA$.

Dans chaque cas, le dispositif tire ses avantages des caractéristiques de conduction électrique de la couche de silice qui doit posséder un fort degré de reproductibilité, de stabilité et d'homogénéité même sous fort champ électrique.

Ces propriétés sont très liées aux conditions d'élaboration des couches. En effet, l'expérience acquise sur les isolants d'épaisseurs supérieures montrent que les paramètres technologiques tels que température d'oxydation, atmosphère d'oxydation, présence d'une étape de préoxydation ont une influence déterminante sur la densité de défauts, l'homogénéité d'épaisseur de la couche ou la structure de la surface qui sont autant de paramètres qui contrôlent les caractéristiques électriques.

En particulier, la présence de composés chlorés durant l'étape d'oxydation s'est avérée bénéfique à bien des égards [5]. Parmi ces composés, le gaz chlorhy-

(*) Communication présentée aux Journées du G.C.I.S., Toulouse les 15 et 16 décembre 1983. drique a été étudié très largement et le tableau I résume ses effets principaux ainsi que les conditions dans lesquelles ils ont été observés.

Le seul effet négatif rapporté dans la littérature est la formation de granulosité à la surface de la couche lorsque la concentration de $\mathrm{HCl}$ dans l'atmosphère oxydante excède $5 \%$ pour une température d'oxydation supérieure à $1100^{\circ} \mathrm{C}$ [6].

Bien que la majeure partie des résultats ait été obtenue relativement à des couches d'épaisseur bien supérieure aux épaisseurs tunnel, on peut raisonnablement attendre une amélioration des propriétés des couches tunnel sous l'influence du gaz chlorhydrique durant l'étape d'oxydation en particulier au niveau de l'homogénéité de l'épaisseur des couches et des défauts.

En effet, il semble peu probable de passiver les ions sodiums du fait de la température élevée nécessaire à cet effet et incompatible avec un bon contrôle de très faibles épaisseurs. Cependant, une diminution très importante de la densité des ions sodium peut être obtenue par un pré-nettoyage $\left(5 \% \mathrm{HCl} / \mathrm{O}_{2}, 1150{ }^{\circ} \mathrm{C}\right.$, $6 \mathrm{~h})$ du tube d'oxydation [12].

L'objet de cet article est de présenter les propriétés des capacités MIS dont la couche de silice d'épaisseur inférieure à $200 \AA$ est obtenue par oxydation thermique dans un mélange $\mathrm{O}_{2} / \mathrm{HCl} / \mathrm{N}_{2}$. L'oxydation en milieu dilué assure un bon contrôle de l'épaisseur de la couche de silice [13] et une température d'oxydation voisine de $950^{\circ} \mathrm{C}$ assure par elle-même une densité de défauts relativement faible [14]. Les paramètres plus 
Tableau I. - Influence du gaz chlorhydrique présent dans l'atmosphère d'oxydation sur les caractéristiques des couches de silice thermique.

[Influence of $\mathrm{HCl}$ during oxidation on the properties of the $\mathrm{SiO}_{2}$ layers.]

\begin{tabular}{|l|c|c|c|}
\hline & $\begin{array}{c}\text { Pourcentage } \\
\text { de } \mathrm{HCl} \text { dans } \\
\text { l'atmosphère } \\
\text { oxydante }\end{array}$ & $\begin{array}{c}\text { Température } \\
\text { d'oxydation }\end{array}$ & Références \\
\hline Diminution des défauts de la couche & $2-3 \%$ & $900-1000^{\circ} \mathrm{C}$ & {$[6]$} \\
\hline $\begin{array}{l}\text { Accroissement de la durée de vie des porteurs dans le } \\
\text { substrat sous-jacent }\end{array}$ & $3 \%$ & $1000{ }^{\circ} \mathrm{C}$ & {$[7]$} \\
\hline $\begin{array}{l}\text { Passivation des ions sodium } \\
\text { Accroissement du champ de claquage }\end{array}$ & $>5 \%$ & $>1100{ }^{\circ} \mathrm{C}$ & {$[8]$} \\
\hline Rugosité superficielle & $>6 \%$ & $1000^{\circ} \mathrm{C}$ & {$[9]$} \\
\hline Diminution de la densité d'états de surface & $0,3 \%$ & $100^{\circ} \mathrm{C}$ & {$[10]$} \\
\hline
\end{tabular}

particulièrement étudiés sont : l'homogénéité de l'épaisseur de la couche de silice, la reproductibilité et l'homogénéité des caractéristiques capacitives et conductives.

\section{Elaboration des structures.}

Les capacités MIS sont obtenues à partir d'un substrat de silicium $\mathrm{P} / \mathrm{P}^{+}$orienté $\langle 100\rangle$, le dopage des deux zones $\mathrm{P}$ étant respectivement de $10^{15} \mathrm{~cm}^{-3}$ et $10^{19} \mathrm{~cm}^{-3}$. Après un nettoyage standard [15] de la surface, celle-ci est oxydée durant $4 \mathrm{~h}$ à $1050^{\circ} \mathrm{C}$ sous oxygène sec. La couche formée est gravée préalablement à la croissance de la couche d'oxyde tunnel qui est menée à $950^{\circ} \mathrm{C}$ dans un mélange $\mathrm{O}_{2}(10 \%) / \mathrm{N}_{2}$ ou $\mathrm{O}_{2}(10 \%) / \mathrm{HCl}(0,2 \%) / \mathrm{N}_{2}$. L'échantillon est ensuite maintenu durant 15 minutes à la température d'oxydation sous azote avant la sortie lente du four. Une couche de chrome ou d'aluminium est enfin déposée sur la face avant. Des dispositifs ayant des surfaces égales à $1600(\mu \mathrm{m})^{2}, 10^{4}(\mu \mathrm{m})^{2}, 9 \times 10^{4}(\mu \mathrm{m})^{2}$ sont disponibles.

\section{Homogénéité d'épaisseur des couches.}

L'épaisseur $D_{\mathrm{ox}}$ des couches est mesurée à partir de la capacité haute fréquence en forte accumulation. Une seconde évaluation est obtenue à l'aide des courbes courant-tension $J-V$ en polarisation directe lorsque l'épaisseur est inférieure à $100 \AA$. Dans ce cas, $D_{\text {ox }}$ est obtenue par comparaison entre courbes $J-V$ expérimentale et théorique [16]. Les paramètres $k_{1}$ et $k_{2}$ de la loi de conduction $\left({ }^{1}\right)$ :

$$
J=k_{1} E_{\text {ox }} \exp \left(-k_{2} / E_{\text {ox }}\right)
$$

( $\left.{ }^{1}\right)$ Dans la relation (1) $E_{\text {ox }}$ représente le champ électrique dans la couche isolante. qui dépendent de la masse effective des électrons dans l'isolant et de la hauteur de barrière métal-isolant sont obtenus pour chaque lot par comparaison des courbes $J-V$ expérimentales et théoriques de dispositifs situés au centre de la plaquette de silicium et pour lesquels l'épaisseur a été évaluée par la première méthode.

Comme le montre la figure 1, la grande sensibilité du courant à l'épaisseur de la couche isolante permet une évaluation précise de cette dernière au moyen des courbes $J-V$.

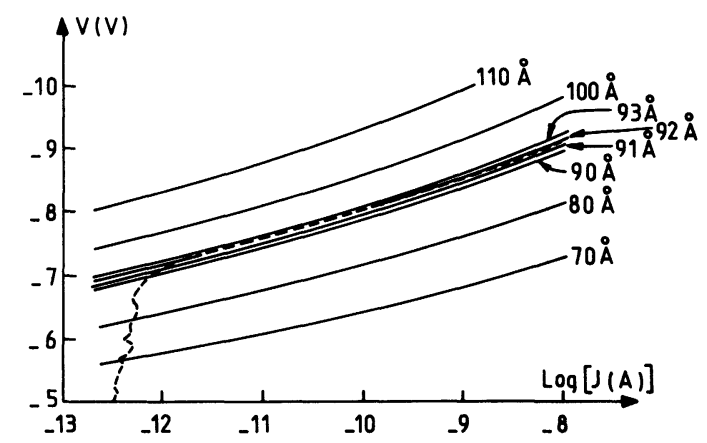

Fig. 1. - Courbes courant $(J)$-tension $(V)$ théoriques, (--- exemple des résultats expérimentaux).

[Theoretical current-voltage curves, (--- example of experimental results).]

Dans le cas d'épaisseurs supérieures à $100 \AA$, ce deuxième type d'évaluation est rendu impossible du fait d'une non-proportionnalité du courant à la surface du dispositif [17].

Par ailleurs, pour certains dispositifs, en nombre relativement restreint, l'épaisseur mesurée par capacité s'est écartée dans la limite de $7 \%$ du comportement général décrit ci-après. Compte tenu du comportement conductif de ces dispositifs, cet écart est à 
attribuer plutôt à des difficultés de prise de contact sous pointe qu'à une réelle dispersion de l'épaisseur.

Les résultats typiques obtenus sur des couches de silice non chlorée sont représentés dans le cas d'une épaisseur voisine de $90 \AA$ sur la figure 2 qui met en évidence une dispersion importante de l'épaisseur et en particulier un accroissement de 5 à $6 \AA$ du centre vers la périphérie de la plaquette.

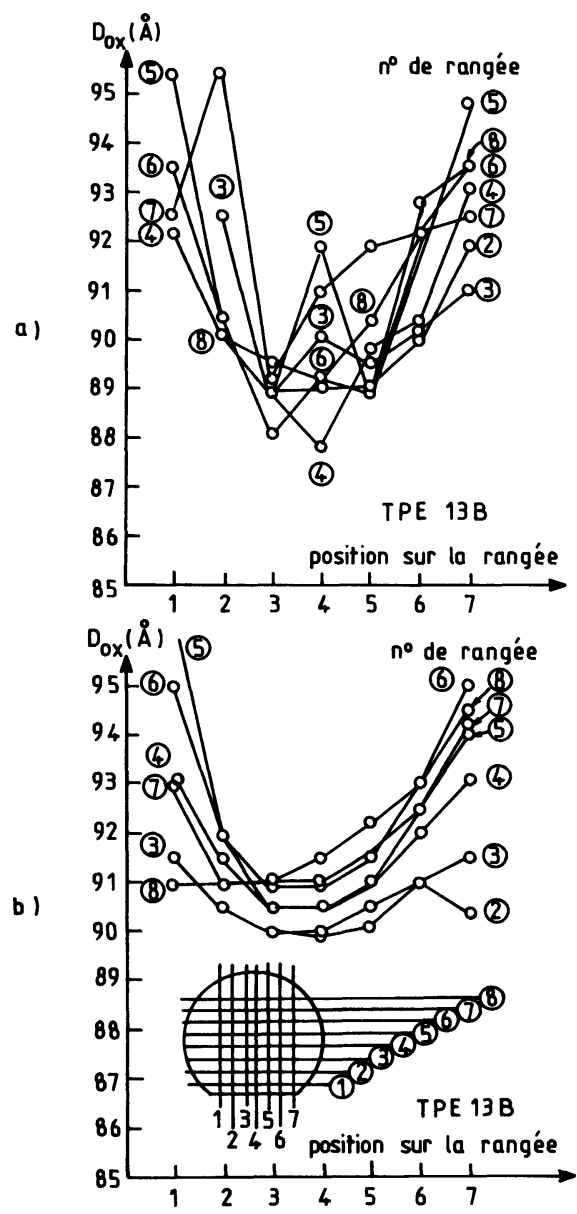

Fig. 2. - Epaisseur en fonction de la position sur la plaquette. Chaque point représente une moyenne sur trois structures différentes. Mesures issues des courbes capacitétension (a) et courant-tension (b).

[Oxide thickness versus position on the wafer, each point represents a mean value taken over three devices, (a) from capacitance-voltage curve, (b) from current-voltage curve.]

On notera, par ailleurs, le bon accord entre les résultats obtenus par les deux méthodes exploitant d'une part les propriétés diélectriques de la couche et d'autre part ses propriétés de conduction. Cet accord valide la méthode de mesure et témoigne d'une bonne homogénéité des propriétés de l'interface $\mathrm{Métal}-\mathrm{SiO}_{2}$.

Dans le cas d'oxydes chlorés les résultats sont représentés sur la figure 3 qui témoigne du fait de l'introduction de chlore dans l'atmosphère oxydante d'une amélioration sensible de l'homogénéité de l'épaisseur quelle que soit sa valeur dans la gamme étudiée.

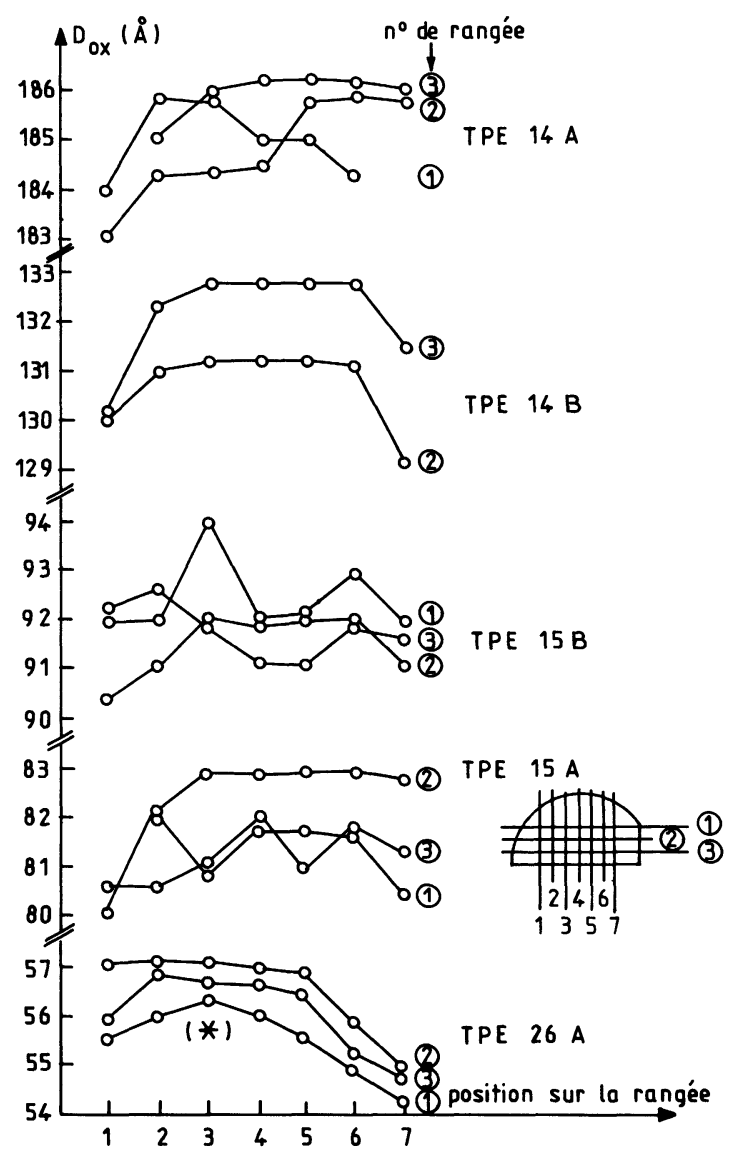

Fig. 3. - Epaisseur en fonction de la position sur la plaquette pour des couches chlorées. Mesures issues de courbes capacité-tension ;

(*) couches obtenues à $900^{\circ} \mathrm{C}$.

[Oxide thickness versus position on the wafer for chlorinated oxide, values obtained from capacitance-voltages curves ;

(*) layers obtained at $900^{\circ} \mathrm{C}$.]

La conséquence de cette amélioration au niveau des caractéristiques courant-tension est représentée sur la figure 4.

La dispersion observée sur la figure 4a est cohérente avec les dispersions en épaisseur observées sur la figure 3 .

L'homogénéité satisfaisante des courbes $J-V$ représentées sur la figure $4 \mathrm{~b}$ témoigne par ailleurs d'une homogénéité de l'ensemble des paramètres contrôlant la conduction à bas niveau à savoir : épaisseur de l'isolant, hauteur de barrière métal-isolant, masse effective des électrons dans la silice.

\section{Cinétique d'oxydation.}

La cinétique de croissance a été relevée dans la gamme d'épaisseur $70 \AA-200 \AA$. Dans cette gamme, elle suit une loi parabolique du type de Deal et Grove [18] indépendamment de la présence ou non de gaz chlo- 

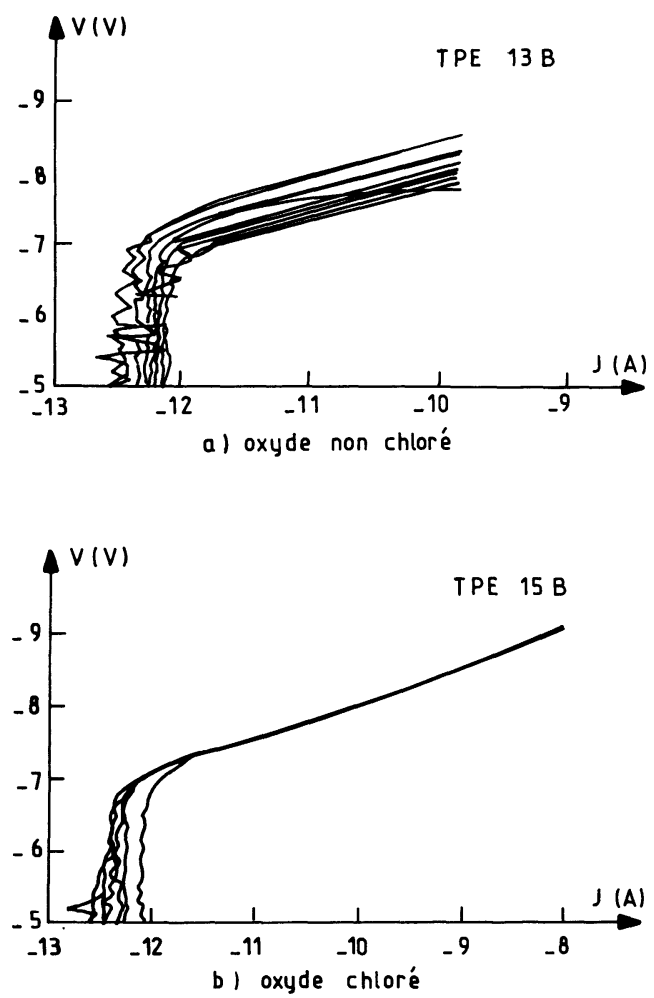

Fig. 4. - Courbes expérimentales courant-tension en polarisation directe.

[Current-voltage curve. Negative on the metal.]

rhydrique. En effet, les courbes de croissance sur la figure 5 peuvent être représentées par les relations :

$D_{\text {ox }}(\AA)=25,6 \cdot t_{\mathrm{ox}}^{0,48} \quad$ pour les oxydes chlorés

$D_{\mathrm{ox}}(\AA)=17,8 \cdot t_{\mathrm{ox}}^{0,48}$ pour les oxydes non chlorés

où $t_{\mathrm{ox}}$ est le temps d'oxydation en minutes.

En l'absence de gaz chlorhydrique et dans les mêmes conditions, Y. Kamigaki et al. [13] ont observé la même loi de croissance bien que la constante de vitesse de réaction parabolique $B$ dans leur cas soit inférieure à celle rapportée ici.

Cependant, si à $1100^{\circ} \mathrm{C} B$ varie sensiblement comme la pression partielle d'oxygène, $B(1 \mathrm{~atm}$. $) / B$ $(0,1 \mathrm{~atm}.) \approx 10$ conformément à la loi de Grove, à

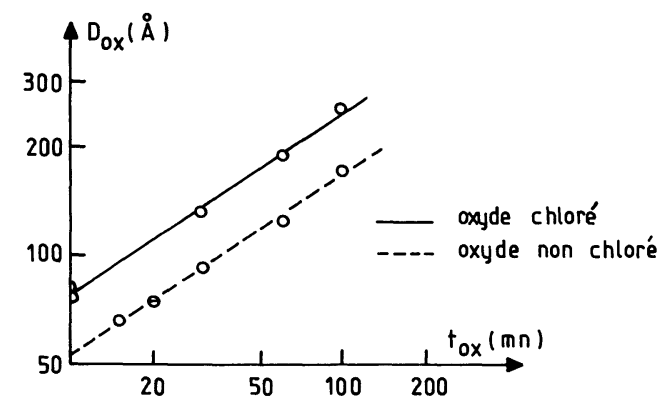

Fig. 5. - Epaisseur $D_{\mathrm{ox}}$ en fonction du temps d'oxydation $t_{\mathrm{ox}}$. [Oxide thickness versus oxidation time.] $950^{\circ} \mathrm{C}$, ce rapport $\left({ }^{2}\right)$ est voisin de 33 tendant à montrer que, à basse température et basse pression, la loi de Henry ne s'applique pas directement. Cependant, une analyse plus détaillée s'avère nécessaire.

En ce qui concerne l'influence du gaz chlorhydrique, les résultats représentés sur la figure 5 montrent que la loi de variation de l'épaisseur en fonction du temps reste du type de Deal et Grove mais que la constante $B$ est approximativement multipliée par deux. Cet accroissement de la vitesse d'oxydation du fait de la présence de gaz chlorhydrique est sensiblement supérieur à celui enregistré par $\mathrm{D}$. W. Hess et al. [19] dans un mélange $\mathrm{HCl}(2 \%) / \mathrm{O}_{2}$ à pression atmosphérique.

\section{Propriétés d'interface.}

Il est maintenant bien établi que les propriétés de l'interface $\mathrm{SiO}_{2}-\mathrm{Si}$ et en particulier la répartition des états de surface sont contrôlées par l'état de la surface du silicium et les conditions d'oxydation.

Il a, par ailleurs, été montré que la présence de gaz chlorhydrique dans l'atmosphère oxydante [11] ou lors d'une étape de pré-nettoyage du four d'oxydation [12] conduit à une réduction très importante de la densité des états $N_{\text {ss }}$ dans le cas d'oxydes épais.

La figure 6 montre l'influence du gaz chlorhydrique présent dans l'atmosphère d'oxydation dans le cas de couches minces de silice. On observe une réduction de $N_{\text {ss }}$ d'un facteur voisin de 3 alors que la répartition énergétique des états n'est pratiquement pas affectée.

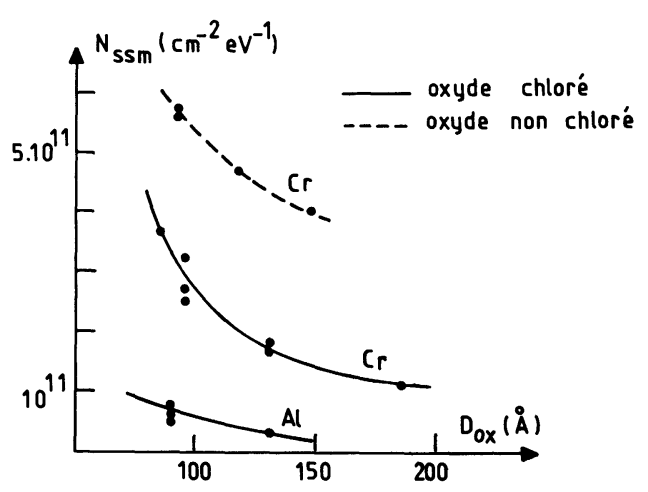

Fig. 6. - Densité maximum d'états de surface $N_{\text {ssm }}$ en fonction de l'épaisseur de la couche d'oxyde, du métal utilisé et de la présence de gaz chlorhydrique.

[Maximum surface state density versus oxide thickness and metal.]

Par ailleurs, cette figure montre que comme dans le cas des couches de silice d'épaisseur inférieure à $40 \AA ̊[20]$ la densité d'états de surface reste très dépendante du métal d'électrode utilisé. En particulier, une

${ }^{2}$ ) La valeur de $B$ (1 atm.) pour une température de $950^{\circ} \mathrm{C}$ a été mesurée dans la gamme d'épaisseur $100 \AA$ $300 \AA$. 
métallisation à l'aluminium conduit à une densité d'état de surface relativement basse compte tenu de l'absence de recuit haute température.

Cependant, ce type de métallisation induit une dispersion très importante des caractéristiques électriques et des champs de claquage inférieurs à ceux observés sur les structures métallisées au chrome [17].

\section{Conclusion.}

L'influence de la présence de gaz chlorhydrique durant l'étape d'oxydation du silicium sous faible pression partielle d'oxygène et à température intermédiaire sur les propriétés électriques des dispositifs MIS associés a été examinée. Il a été montré que la cinétique de croissance n'est pas fondamentalement modifiée et que par conséquent le mécanisme d'oxydation n'apparaît pas altéré par l'atmosphère chlorée.

La meilleure homogénéité de l'épaisseur des couches ne peut être imputée à la quantité d'eau générée lors de la réaction :

$$
\mathrm{HCl}+\mathrm{O}_{2} \rightleftarrows \mathrm{H}_{2} \mathrm{O}+\mathrm{Cl}_{2} \text {. }
$$

En effet, ce même effet a été observé lors d'une oxydation en ambiance humide chlorée à pression atmosphérique dans la gamme d'épaisseur (800$900 \AA \AA)$ [21]. Plus vraisemblablement, le gaz chlorhydrique pourrait jouer le rôle de passivant pour des défauts ou impuretés situés à la périphérie de la plaquette lors des premiers stades de l'oxydation où la cinétique est contrôlée par la réaction de la surface.

Par ailleurs, la reproductibilité de l'épaisseur moyenne d'un lot à l'autre s'est avérée satisfaisante à l'exception des plus faibles épaisseurs. Dans ce cas, ceci peut être imputé au manque de contrôle de la durée d'oxydation du fait des faibles temps d'oxydation et de l'existence d'un temps d'établissement du flux oxydant dans le tube d'oxydation.

L'effet du gaz chlorhydrique a une incidence positive importante au niveau des caractéristiques courant-tension sans cependant avoir d'effet sensible sur la dispersion des champs de claquage à forte injection [17].

\section{Remerciements.}

Les auteurs tiennent à remercier les membres de la Centrale de Technologie du L.A.A.S. pour la réalisation des dispositifs.

\section{Bibliographie}

[1] Johnson, W. S., Kuhn, G. L., Renninger, A. L. and Perlagos, G., Electronics 28 (1980) 113.

[2] Sarrabayrouse, G., Buxo, J., Sebaa, J. P. and Essaid, A., I.E.E. Proc. part I, 128 n $^{\circ} 2$ (1981) 53.

[3] Augusta, B., Proceedings of the International Conference on large scale integration, Paris, December 3-6, 1974, p. 226-232.

[4] Stirn, R. J. and YeH, Y. C., Appl. Phys. Lett. $27 \mathrm{n}^{\circ} 2$ 1075.

[5] Singh, B. R. and BALK, P., J. Electrochem. Soc. 125 no 3 (1978) 453.

[6] Itsumi, M. and Kiyosumi, F., Appl. Phys. Lett. 40 no 6 (1982) 496.

[7] Young, D. R. and Osburn, C. M., J. Electrochem. Soc. 170 (1973) 1578.

[8] Monkowski, J., STACH, J. and TresSer, R. E., J. Electrochem. Soc. 126 (1979) 1129.

[9] Osburn, C. M., J. Electrochem. Soc. 121 (1974) 809.

[10] Hirabayashi, K. and Iwamura, J., J. Electrochem. Soc. 120 (1973) 1595.
[11] Severi, M. and Soncini, G., Electron. Lett. 8(1972) 402.

[12] Hamasaki, M., J. Appl. Phys. 52 (no 5) (1981) 3484.

[13] Kamigaki, Y. and Itoh, Y., J. Appl. Phys. 48 n $^{\circ} 7$ (1977) 2891.

[14] Osburn, C. M., J. Electrochem. Soc. 121 (1974) 809.

[15] Amick, J. A., Solid State Technol. 47 (1976).

[16] Capilla, J., Thèse de Doctorat de 3e Cycle, no 2910, Université Paul Sabatier, Toulouse, 1983.

[17] Capilla, J. and Sarrabayrouse, G., Rev. Phys. Appl. 19 (1984) 343.

[18] Deal, B. E. and Grove, A. S., J. Appl. Phys. 36 (1965) 3770.

[19] HeSs, D. W. and DeAL, B. E., J. Electrochem. Soc. 124 $\mathrm{n}^{\circ} 5$ (1977) 735.

[20] KaR, S. and Dahlke, W. E., Solid State Electron. 15 (1972) 221.

[21] Ahlgren, D. C., Proceedings of the 4th International Symposium on Silicon Materials Science and Technology, 11-15 mai 1981, Minneapolis, p. 832. 